\title{
Fabrication of Extremely Short Length Fiber Bragg Gratings for Sensor Applications
}

\author{
Meng-Chou Wu, Robert S. Rogowski, and Ken K. Tedjojuwono \\ NASA Langley Research Center \\ Hampton, Virginia, USA \\ m.c.wu@larc.nasa.gov
}

\begin{abstract}
A new technique and a physical model for writing extremely short length Bragg gratings in optical fibers have been developed. The model describes the effects of diffraction on the spatial spectra and therefore, the wavelength spectra of the Bragg gratings. Using an interferometric technique and a variable aperture, short gratings of various lengths and center wavelengths were written in optical fibers. By selecting the related parameters, the Bragg gratings with typical length of several hundred microns and bandwidth of several nanometers can be obtained. These short gratings can be apodized with selected diffraction patterns and hence their broadband spectra have a welldefined bell shape. They are suitable for use as miniaturized distributed strain sensors, which have broad applications to aerospace research and industry as well.
\end{abstract}

\section{Keywords}

Fiber Bragg gratings; Optical fiber sensors; Apodization.

\section{INTRODUCTION}

Optical fiber Bragg gratings (FBG's) have a broad range of application from wave filters, reflectors, and fiber amplifiers for telecommunications to Bragg grating sensors, for sensing. The application of FBG's to strain measurements has been of great interest to industries. Fiber optic stain sensors, compared to conventional strain gauges, have similar sensitivities, but are much lighter weight and require simpler wiring. These advantages are especially important to aerospace applications. However, as strain sensors encounter micro-strains-where the strained area is smaller than the dimensions of the sensors-strain gauges average over their finite area and are therefore insensitive to the variation of micro-strains. In contrast, the spectra of Bragg grating sensors are distorted by micro-strains, which may complicate strain measurements. Bragg gratings with shorter length certainly can avoid this problem. The very short gratings also allow the sensors to be miniaturized. The questions are then: How does the width of spectra change with the length of gratings? Are the broadened spectra resulting from the shorter length still suitable for the strain measurements?

For some applications of FBG other than strain sensors, fiber Bragg gratings with broader spectra are required, for example, a broadband reflector with a typical bandwidth of several $\mathrm{nm}$. It is of interest to ask if short Bragg gratings can be created to contain well-defined spectra with the same order of bandwidth.

This paper presents a study of diffraction effects on both the spatial and wavelength spectra of fiber Bragg gratings and a new technique to fabricate extremely short Bragg gratings in optical fibers with well-defined spectra for various applications

\section{THEORIES}

\section{Inscription and Properties of Bragg Gratings}

In general, as a photosensitive fiber is exposed to a pair of interfering ultraviolet (UV) laser beams, a Bragg grating is created along the fiber core. The resulting periodic changes of refractive index along the fiber core sets the Bragg condition for wave propagation in the fiber. This is equivalent to a Bragg wavelength $\lambda_{\mathrm{B}}$. The length of interference pattern created by the laser beams determines the length of Bragg grating and thereby the line width centered at $\lambda_{\mathrm{B}}$. In general, the spectrum of the reflected light from, or transmitted through a Bragg grating is narrower as the length of grating is longer, and vice versa. For example, the Bragg gratings used for creating fiber lasers have a length, typically of tens of $\mathrm{mm}$ and those used for strain sensors are least several $\mathrm{mm}$ long to maintain a line width of a couple tenths of $\mathrm{nm}$.

There are two major techniques used to write Bragg gratings in optical fibers. The first uses of a phase mask, which is a UV transparent plate with etched surface grooves, resembling a grating of specific period [1]. As the UV laser beam passes through the mask it is diffracted into various orders. The pair of first-order diffracted beams, normally containing most of the laser energy, forms an interference pattern right behind the mask. An optical fiber is placed in that area to get masked and thereby inscribed with the Bragg grating. This technique is simple for a well-defined Bragg wavelength. However, to write gratings of various wavelengths requires various phase masks.

Another technique involves an optical system, splitting the laser beam and steering the two split beams to interfere with each other, as shown schematically in Figure 1. This type of set-up is basically an interferometer $[2,3]$. This technique is therefore referred to as an interferometric method. The angle between the two interfering beams, now 
adjustable, determines the period of the interference fringes and thereby the grating, as the fiber is placed in the interference region.

For a Bragg grating with a length $\mathrm{L}$, the bandwidth of its reflection spectrum, defined as the full width between two first minimums, is given approximately by [4]

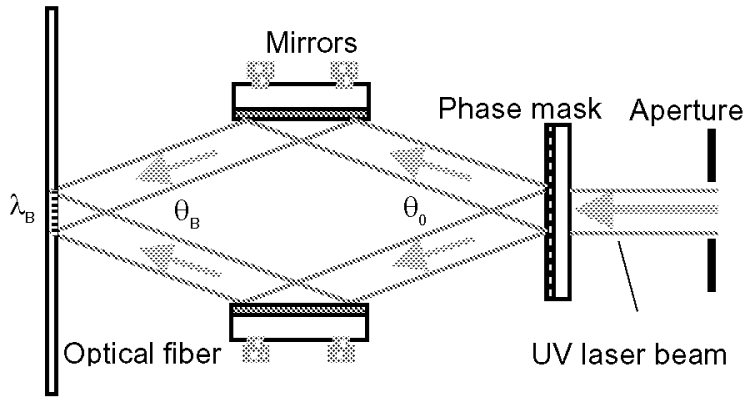

Figure 1. A schematic representing the interferometric technique for generating fiber Bragg gratings. The Bragg wavelength $\lambda_{B}$ is determined by the adjustable $\theta_{\mathrm{B}}$

$$
\Delta \lambda=\frac{\lambda_{B}{ }^{2}}{\pi L n_{e f f}}\left(\kappa^{2} L^{2}+\pi^{2}\right)^{1 / 2}
$$

where $n_{\text {eff }}$ is the effective refractive index of the fiber core, and $\kappa$, the coupling coefficient, is on the order of $\Delta n / \lambda$, and $\Delta \mathrm{n}$ is the amplitude of the periodic change of refractive index along the fiber core.

For a finite length grating with a weak coupling coefficient, i.e., $\kappa \mathrm{L} \ll 1$, Eq. (1) is reduced to

$$
\Delta \lambda=\frac{\lambda_{B}^{2}}{n_{e f f} L} .
$$

This expression states that the width of the wavelength spectrum for a grating is inversely proportional to its spatial length. However using a simple number $\mathrm{L}$ to describe the spectrum is assuming the $\Delta \mathrm{n}$ is more or less uniform along the length. In some cases a fine structure of the profile of $\Delta \mathrm{n}$ needs to be considered.

\section{Diffraction Effects on Bragg Gratings}

In practice, an aperture or a slit is used to regulate the spot size of the UV laser beam in order to get the desired grating length. However, as the opening of the aperture gets smaller and smaller, diffraction effects become more significant, the spot size of the UV beam, and thereby the grating length are not simply proportional to the linear dimension of the aperture any more.

Not only the aperture size but also the distance of the aperture to the object becomes critical for considering diffraction effects. To investigate the effects, one has to determine if it is the case of far field effect-Fraunhofer diffraction, or the near field one-Fresnel diffraction.

\section{Fraunhofer Diffraction}

Suppose an aperture is normally illuminated by a monochromatic plane wave. Figure 2 describes the diffraction geometry of an aperture. The diffracting aperture, $\mathrm{A}$ is assumed to lie in the $(\xi \eta)$ plane, and is illuminated in the positive $\mathrm{z}$ direction, and $(\mathrm{x}, \mathrm{y})$ is the observation plane, parallel to the $(\xi \eta)$ plane and at normal distance $z$ from it.

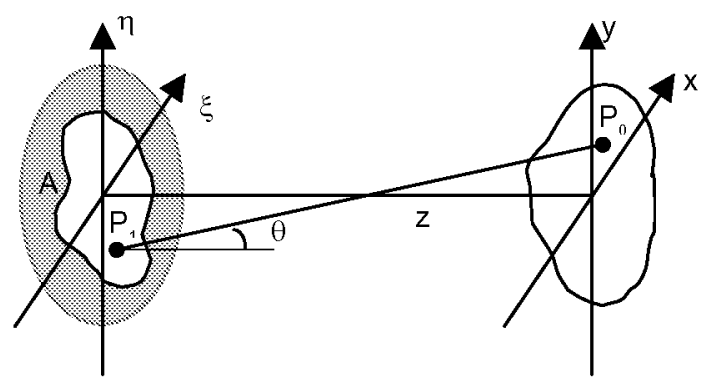

Figure 2. Diffraction geometry of an aperture .

Based on the Huygens-Fresnel principle, the diffraction can be described by the Fresnel-Kirchhoff (F-K) diffraction integral, [5]

$U(x, y)=\frac{z}{j \lambda} \iint_{A} U(\xi, \eta) \frac{\exp \left(j k r_{01}\right)}{r_{01}^{2}} d \xi d \eta$

where $r_{01}$ is the distance from $P_{0}$ to $P_{1}$ and given by

$$
r_{01}=\left[z^{2}+(x-\xi)^{2}+(y-\eta)^{2}\right]^{1 / 2}
$$

To reach this expression, there is an assumption that the observation distance is much larger that the wavelength, i.e., $r_{01} \gg \lambda$. To reduce the F-K Integral to a simpler expression, further approximations can be introduced. The well-known Fraunhofer approximation assumes that

$$
z>\left(\xi^{2}+\eta^{2}\right)_{\max } / \lambda
$$

is satisfied and retains only the first term of the binomial expansion of $r_{01}$, Eq. (4).

For the case of a single slit with a width of $b$, this condition can be expressed as $z>b^{2} / \lambda$. The solution of the F-K integral, using Eq. (3), contains a sinc function and the intensity of the diffraction pattern can be expressed as

$I(x)=|U(x)|^{2}=I_{0}(\sin \beta / \beta)^{2}$,

where $\beta=1 / 2 \mathrm{~kb} \sin \theta$, and $\mathrm{k}$ is the wavenumber. This is the well-known Fraunhofer diffraction pattern.

The first minimun occurs for $\beta=\pi$, i.e., $\sin \theta=\lambda / b$. If the directional angle, $\theta$ is assumed to be small, the width of the 
central lobe of the diffraction pattern can then be expressed as $\Delta \mathrm{W}=2 \mathrm{z} \sin \theta=2 \mathrm{z} \lambda / \mathrm{b}$. Thus it varies inversely with the slit width. For the ongoing and later discussion, it is convenient to define a factor,

$$
M_{F}=\Delta W / b=2 z \lambda / b^{2}
$$

which can be interpreted as a magnification. The Fraunhofer approximation requires that $\mathrm{M}_{\mathrm{F}} \gg 1$. Hence the Fraunhofer diffraction pattern becomes much wider than the size of the aperture. It seems ironic that if one pursues using the aperture to reduce the spot size of the laser beam the opposite occurs when Fraunhofer diffraction is in effect.

However the conditions required for Fraunhofer diffraction are severe. In a practical situation, for example, at a wavelength of $0.25 \mu \mathrm{m}$ (an Excimer laser beam) and an aperture width of $\mathrm{b}=250 \mu \mathrm{m}$, and a point of observation at $\mathrm{z}=$ $250 \mathrm{~mm}$. The ratio of $\mathrm{z} /\left(\mathrm{b}^{2} / \lambda\right)=1$. At this distance, $\mathrm{z}$ is not much greater than $b^{2} / \lambda$. There is another alternative, less stringent condition for a valid Fraunhofer approximation, known as the "antenna designer's formula", which only requires that $\mathrm{z}>2 \mathrm{D}^{2} / \lambda$, where $\mathrm{D}$ is the linear dimension of the aperture. However, for the near field diffraction patterns, one should consider the more general Fresnel Diffraction.

\section{Fresnel Diffraction}

The Fresnel approximation retains the first two terms of the binomial expansion of $r_{01}$, Eq. (4). Neglecting the higher order terms, the condition requires that

$$
z^{3}>>\frac{\pi}{4 \lambda}\left[(x-\xi)^{2}+(y-\eta)^{2}\right]_{\max }^{2}
$$

Actually the Fresnel approximation yields highly accurate results even at distances that are very close to the aperture [6].

Suppose a square aperture of width $\mathrm{b}$ is illuminated by a monochromatic plane wave. The F-K diffraction integral can be reduced as

$$
U(x, y)=\frac{e^{j k z}}{j \lambda z} \int_{-b / 2}^{b} \int^{2} \exp \left\{j \frac{\pi}{\lambda z}\left[(x-\xi)^{2}+(y-\eta)^{2}\right]\right\} d \xi d \eta
$$

This integral doesn't have a unique solution, however it can be expressed as tabulated functions of Fresnel integrals, which can be defined as

$C(s)=\int_{0}^{s} \cos \left(\pi t^{2} / 2\right) d t$

and

$$
S(s)=\int_{0}^{s} \sin \left(\pi t^{2} / 2\right) d t .
$$

For further simplicity, considering only a single slit of width $b$, the intensity of the wave field is therefore given by

$$
\left.I(x)=\frac{1}{2}\left\{C\left(\alpha_{2}\right)-C\left(\alpha_{1}\right)\right]^{2}+\left[S\left(\alpha_{2}\right)-S\left(\alpha_{1}\right)\right]^{2}\right\}
$$

where

$\alpha_{1}=-\sqrt{\frac{2}{\lambda z}}\left(\frac{b}{2}+x\right) \quad \alpha_{2}=\sqrt{\frac{2}{\lambda z}}\left(\frac{b}{2}-x\right)$.

Figure 3 shows a series of graphs representing the Fresnel diffraction intensity distribution along the $\mathrm{x}$-axis for various slit widths, $b$ at fixed distance, $z$, and fixed wavelength, $\lambda$. To simplify the description, it is convenient to define the Fresnel number,

$N_{F}=\frac{(b / 2)^{2}}{\lambda z}$,

and the normalized distance variable, $u=x /(\lambda z)^{1 / 2}$. It should be noted that the comparison for fixed $b$ and various $\mathrm{z}$ is the same.

It is interesting to see that, as the $\mathrm{N}_{\mathrm{F}}$ becomes large (large $\mathrm{b}$ or small z) the diffraction pattern is shaped like a top hat, approaching the shape of the aperture itself. As the $\mathrm{N}_{F}$ becomes small (small $b$ or large $\mathrm{z}$ ) the diffraction pattern becomes Fraunhofer-like, and the width of the diffraction pattern is much wider than the actual aperture size. 

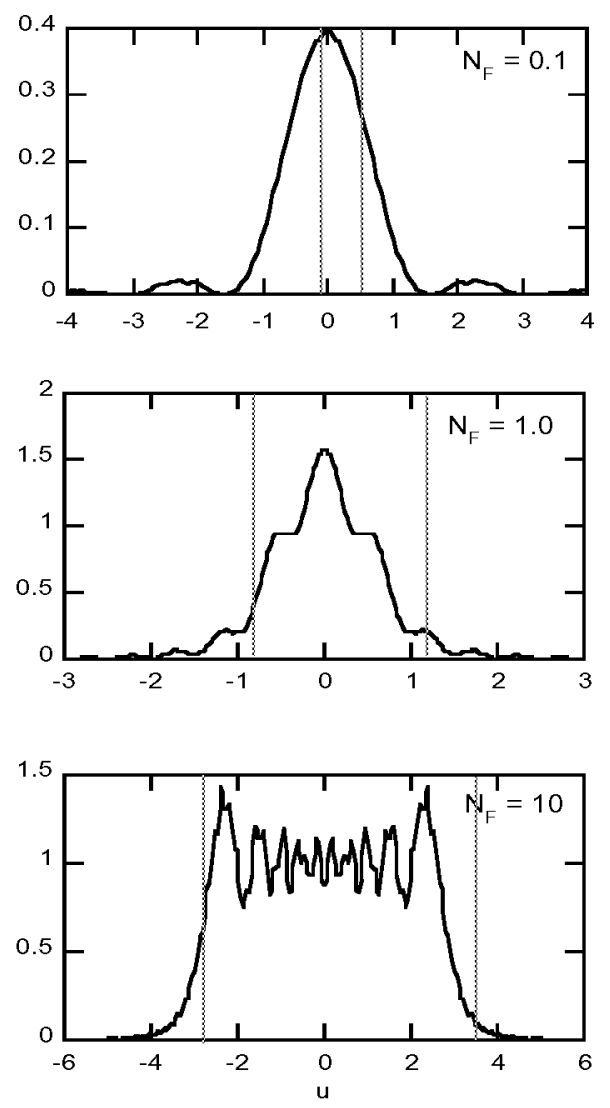

Figure 3. Fresnel diffraction patterns from a single slit of various widths at a fixed distance. Two gray lines in the respective graphs indicate the actual aperture sizes.

\section{APODIZATION}

If an aperture is used for regulating the spot size of a laser beam then the resulting diffraction pattern modulates the interference pattern for writing gratings. In other words, a diffraction pattern determines the physical shape (the spatial spectrum) of a Bragg grating and hence its wavelength spectrum.

For a long grating with the spatial spectrum resembling the shape of a top hat, the wavelength spectrum typically resembles a sinc function. The spatial length of the grating only affects the width of its spectrum, i.e., its linewidth. However as the grating becomes physically shorter, the wavelength spectrum is more complicated. If a welldefined spectrum is the goal for fabricating a Bragg grating then special attention must be paid to shaping the pattern.

Apodization is a conventional technique for shaping the spectrum of a grating. It is well known that the Fourier transform of a top hat function is a sinc function. If the top hat is replaced by a cosine function the resultant Fourier transform is still like a sinc function but with the amplitude of its side lobes substantially reduced [7]. Hence this effect is called apodization. In practice, apodization could be ac- complished, for example, by means of an amplitude-varied mask placed over the aperture [8]. However this technique is more suitable for writing longer, especially chirped gratings with a typical length of several centimeters.

Alternatively for writing short gratings, one can use the diffraction patters of a small $\mathrm{N}_{\mathrm{F}}$ (as $\mathrm{N}_{\mathrm{F}}=0.1$ in Figure 3) with its sinc-like central lobe to shape the gratings. Thus the resultant wavelength spectra are apodized. One can call this self-apodization.

\section{EXPERIMENTAL}

A pulsed Excimer laser of $248 \mathrm{~nm}$ with maximum output of $450 \mathrm{~mJ}$ was used to write all the gratings discussed here. Both standard telecommunication fiber and some borongermanium co-doped photosensitive fiber were used. Typical exposure was a $20 \mathrm{~Hz}$ pulse rate for a duration of less than 5 minutes. For the standard telecommunication fiber only very weak gratings with reflectivities, $\mathrm{R}$, lower than $0.1 \%$ were obtained as expected. For the photosensitive fiber, the short grating length kept the reflectivity low, typically less than $15 \%$. In general very strong gratings with $\mathrm{R}$ $>99 \%$ were avoided. For those high reflectivity gratings, the spectrum would broaden and deviate from a sinc shape, whose width would be otherwise inversely proportional to the grating length.

For varying the wavelength of Bragg gratings, the interferometric technique of writing gratings was employed. A phase mask was used as a beam splitter and a pair of mirrors was used to recombine the two split beams forming an interference pattern. A single slit aperture was placed in front of the phase mask to regulate the width of the incoming laser beam. The width of the slit varied from several millimeters to about $50 \mu \mathrm{m}$.

A Frequency domain demodulation system [9] was used to read out the gratings. The detected signals were further processed (FFT and inverse-FFT) to display both the spatial and wavelength spectra of gratings.

\section{RESULTS}

Figure 4 shows a Bragg grating written in a standard fiber. The slit width was $2 \mathrm{~mm}$. The spatial spectrum of this grating, Figure 4(a), shows the typical features of a top hat. Its wavelength spectrum, Figure 4(b), has the characteristic shape of sinc function. Also shown in the figure is a computed Fresnel diffraction pattern, using all the parameters from actual grating writing (with $\mathrm{N}_{\mathrm{F}}=16.4$ ). A slight offcenter observation position was introduced to simulate the small misalignment in reality. This results in the asymmetric form of the spectrum. This diffraction pattern is characteristically similar to the spatial spectrum of the grating. 

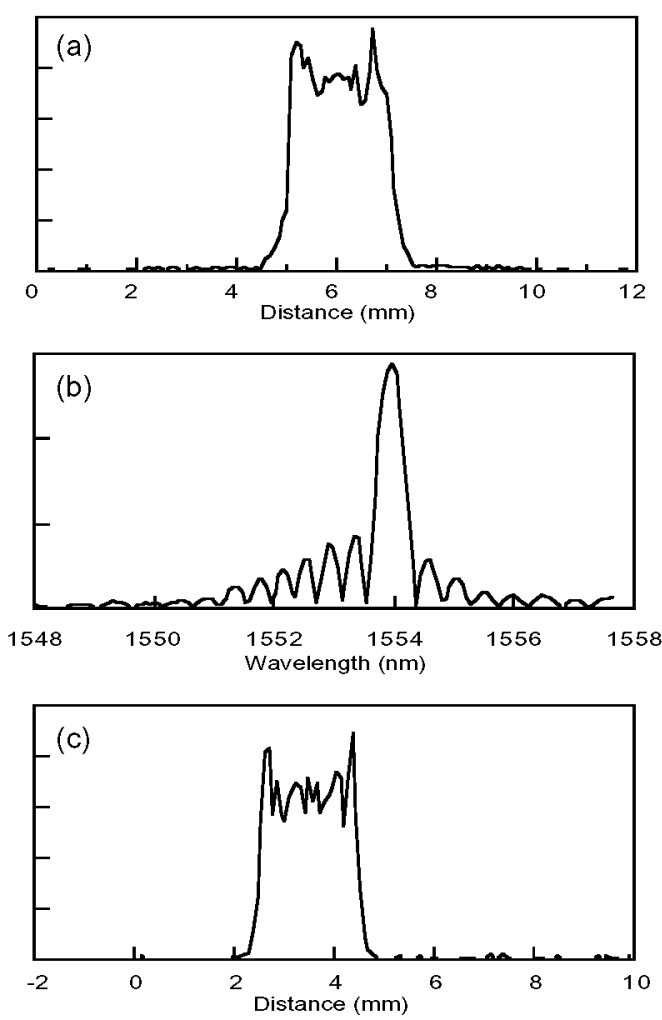

Figure 4. (a) is the wavelength spectrum and (b) the spatial spectrum of a written Bragg grating with a slit width of $2 \mathrm{~mm}$. Using the same parameters, a Fresnel diffraction pattern is calculated and shown in (c).
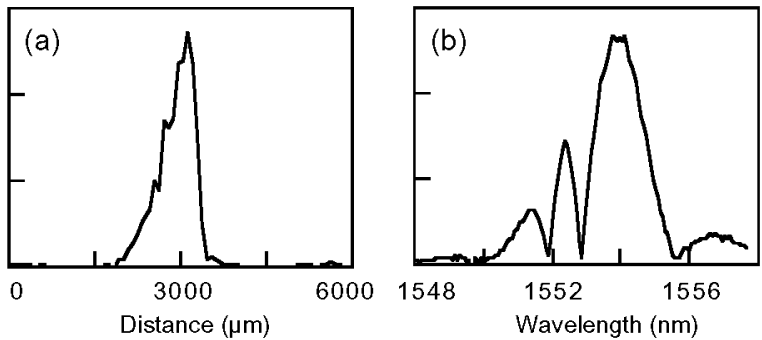

Figure 5. The spatial (a) and wavelength (b) spectra of a Bragg grating written at a distance, $z$, of $0.2 \mathrm{~m}$ and $a$ slit width, b, of $700 \mu \mathrm{m}$ (with $N_{F}=2.5$ ).

As the previous discussion stated, simply reducing the aperture size to write short gratings will encounter the complication of the wavelength spectra. Figure 5 shows the spatial and wavelength spectra of a Bragg grating, written with a slit size, $b$, of $700 \mu \mathrm{m}$ at a distance, $\mathrm{z}$, of $0.2 \mathrm{~m}$ (with $\mathrm{N}_{\mathrm{F}}=2.5$ ). Apparently the wavelength spectrum, Figure 5(b) contains large side lobes.

The spectra of a series of short length gratings are shown in Figure 6 . These gratings were written at a fixed distance $(\mathrm{z}$ $=0.246 \mu \mathrm{m}$ ) with aperture size, $\mathrm{b}$, changing from $400 \mu \mathrm{m}$ down to $50 \mu \mathrm{m}$. Their equivalent $\mathrm{N}_{\mathrm{F}}$ 's and $\mathrm{M}_{\mathrm{F}}$ 's are given in Table 1.

Table 1. The calculated Fresnel number, $N_{F}$, and the magnification number, $M_{F}$, for a series of written Bragg gratings as shown in Figure 6.

\begin{tabular}{c|cccccc}
\hline $\begin{array}{c}b \\
(\mu \mathrm{m})\end{array}$ & 400 & 300 & 200 & 150 & 100 & 50 \\
\hline $\mathrm{N}_{\mathrm{F}}$ & 0.66 & 0.37 & 0.16 & 0.09 & 0.04 & 0.01 \\
& & & & & & \\
$\mathrm{M}_{\mathrm{F}}$ & 0.73 & 1.36 & 3.05 & 5.42 & 12.2 & 48.8 \\
\hline \multicolumn{5}{c}{$\mathrm{z}=0.246 \mathrm{~m}$ and $\lambda=0.248 \mu \mathrm{m}$}
\end{tabular}

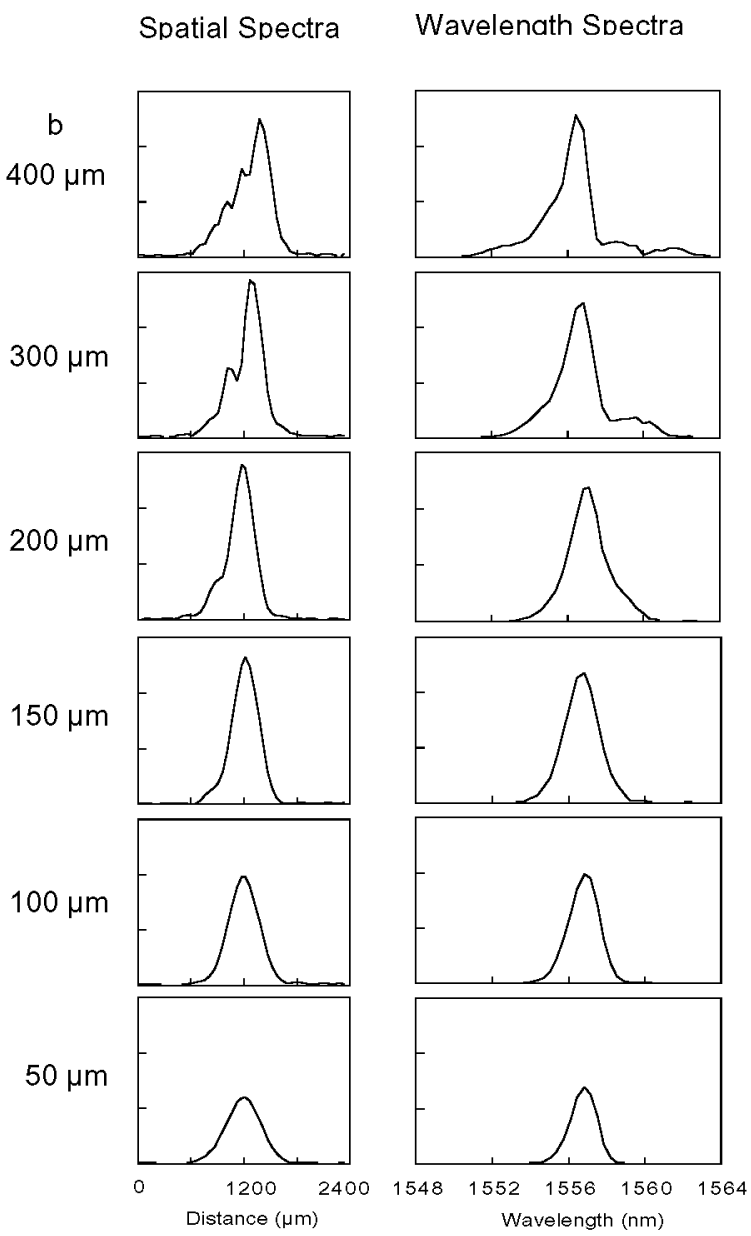

Figure 6. A series of fiber Bragg gratings written at a fixed distance, $z$, and various slit widths, b. Shown in the left column are the spatial spectra and in the right their respective wavelength spectra.

It is clear that at large $N_{F}$ the wavelength spectrum of a particular grating still has some side lobe because of its less 
well-defined spatial spectrum. At smaller $\mathrm{N}_{F}$ (for $\mathrm{b}$ less than $300 \mu \mathrm{m}$ ), the spatial spectrum of a grating becomes smoother and more like a sinc function. Its wavelength spectrum is hence apodized, with the side lobes well suppressed.

Shown in Figure 7 are the spectra of another series of gratings written at a constant aperture size $(\mathrm{b}=100 \mu \mathrm{m})$ with various distances $z$. Their equivalent $N_{F}$ 's are in the range of 0.029 to 0.069 . The relatively small $\mathrm{N}_{\mathrm{F}}$ for each grating keeps its respective wavelength spectrum a smooth bell shape. However, for a larger $\mathrm{z}$, the width of the spatial spectrum is broader (and the wavelength spectrum narrower.) This broadening of spatial spectra can also be easily seen with its respective $\mathrm{M}_{\mathrm{F}}$, ranging from 7.3 to 17.2 . The larger $M_{F}$ is the broader the spatial spectrum.
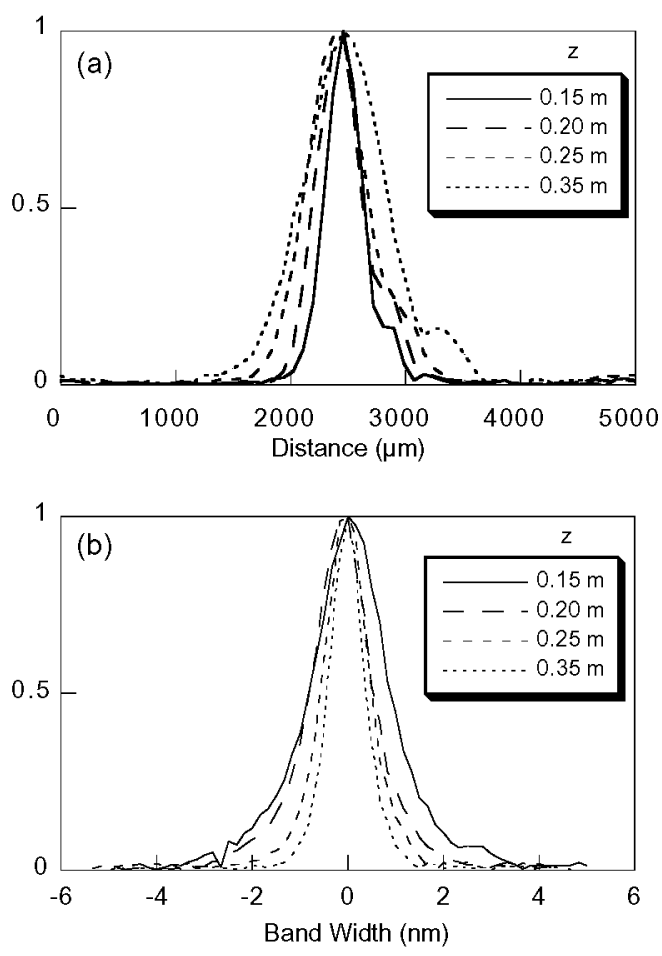

Figure 7. A series of fiber Bragg gratings written at various distances, $z$, and a fixed slit width, b. Shown in (a) are the spatial spectra and in (b) their respective wavelenath spectra.

\section{DISCUSSION}

Suppose that the goal, as mentioned previously, is to fabricate Bragg gratings that have spatial lengths as small as possible and at same time have the well-defined, selfapodized wavelength spectra. Then from the analysis of the Fresnel diffraction patterns and the above experimental results one can set up a kind of criterion such that the goal can be reached.

Although there is no sharp transition for the characteristic changes of the spectra resulting from varying $\mathrm{N}_{\mathrm{F}}$, it is ob- vious, to obtain satisfactory self-apodized gratings, one can keep the $\mathrm{N}_{\mathrm{F}}$ smaller than 0.25 , i.e.,

$N_{F}<0.25$.

Since $M_{F}=1 / 2 N_{F}$. The above condition is equivalent to

$M_{F}>2$

or $z>b^{2} / \lambda$. This is very close to the "antenna designer's formula", though slightly less stringent. From the previous section on apodization, it is stated that in order to produce self-apodized gratings, the diffraction pattern needs to be Fraunhofer-like

Now some formulae developed in the discussion of Fraunhofer diffraction can be used here. Since $M_{F}=\Delta W / b$, Eq. (15) reduces to

$\Delta W>2 b$.

The physical length (spatial width) of a self-apodized grating will be broader than the width of aperture that is used to create the grating

On the other hand, keeping $\Delta \mathrm{W}$ small is also the goal to keep the sensor size small. Then from Eq. (16), b needs to be small. However, since $\Delta \mathrm{W}=2 \lambda_{\mathrm{z}} / \mathrm{b}$, in order to keep both the $\Delta \mathrm{W}$ and $\mathrm{b}$ small, $\mathrm{z}$ must be small at the same time (and still meet the criterion). Therefore, using the smaller aperture size to make shorter length self-apodized gratings one needs to bring the aperture closer to the object.

Certainly there is a physical limitation for pushing $\Delta \mathrm{W}$ to be small. First, using the interferometric technique to write gratings requires a minimum length of the optics behind the aperture, and thereby a lower limit for z. Secondly, the periodicity in $\Delta \mathrm{n}$ for most Bragg gratings discussed here is on the order of $1 \mu \mathrm{m}$. If a grating only contains a few periods it becomes too weak to be measurable and usable.

\section{APPLICATIONS}

As stated in previous sections, these short length fiber Bragg gratings with well-defined spectra apparently can be used as strain sensors just like ordinary length gratings. Their physical dimension is an advantage compared to the latter in some applications requiring short length sensors.

In Figure 8, shown is an example of a miniature distributed strain sensing device, using short length gratings. An optical fiber inscribed with 16 Bragg gratings was bonded to a piece of micro mechanical sensor. The fiber runs across both sides of the $\mathrm{H}$-shape bridge, which is $3 \mathrm{~cm}$ long. The gap distance forming the $\mathrm{H}$-shape is $500 \mu \mathrm{m}$, which allows the bridge to move in plane. This design is intended to measure the tangential force. The details and results will be presented in other studies.

\section{SUMMARY}

This study has developed a new technique to fabricate extremely short length fiber Bragg gratings which are suitable 
for distributed strain sensing. The spatial width of the gratings can be as small as a few hundred microns. This type of grating allows the sensor system to be miniaturized. A simple formula has also been developed to give the guideline of controlling related parameters in order to fabricate short length gratings with self-apodized spectra.

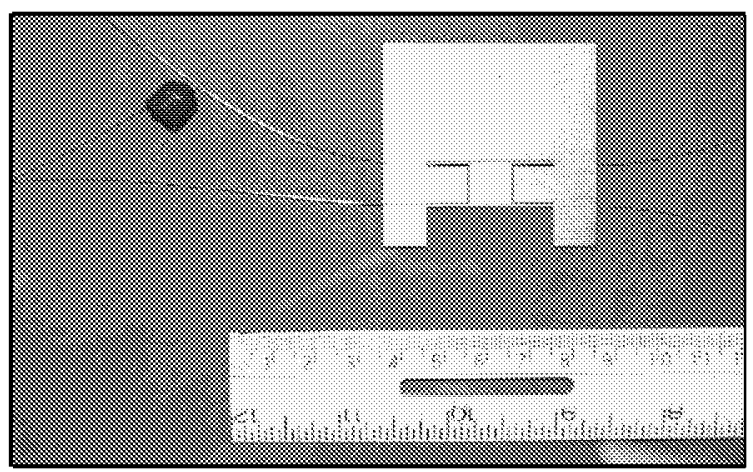

Figure 8. A miniature distributed strain sensor. An optical fiber written with short length Bragg gratings is bonded to a piece of micro mechanical sensor.

\section{REFERENCES}

[1] K. O. Hill, B. Malo, F. Bilodeau, D. C. Johnson, and J. Albert, "Bragg gratings fabricated in monomode pho- tosensitive optical fiber by UV exposure through a phase mask," Appl. Phys. Lett., 62, 1035 (1993).

[2] G. W. Meltz, W. Morey, and W. H. Glenn, "Formation of Bragg gratings in optical fibers by a transverse holographic method," Opt. Lett., 14, 823 (1089).

[3] P. E. Dyer, R.J. Farley, and R. Giedl, "Analysis and application of a 0/1 order Talbot interferometer for 193 nm laser grating formation," Opt. Comm., 129, 98 (1996)

[4] A. Ghatak and K. Thyagarajan, Introduction to Fiber Optics, Cambridge University, Cambridge (1998).

[5] J. W. Goodman, Introduction to Fourier Optics, $2^{\text {nd }}$ ed., McGraw-Hill, New York (1996).

[6] S. J. Mihailov, F. Bilodeau, K. O. Hill, D. C. Johnson, J. Albert, and A. S. Holmes, "Apodization technique for fiber grating fabrication with a halftone transmission amplitude mask," Appl. Opt., 39, 3670 (2000).

[7] W. H. Southwell, "Validity of the Fresnel approximation in the near field," J. Opt. Soc. Am., Vol, 71, 7 (1981).

[8] G. R. Fowlers, Introduction to Modern Optics, $2^{\text {nd }}$ ed., Dover Publications, New York (1975).

[9] M. Froggatt and J. Moore, "Distributed measurement of static strain in an optical fiber with multiple Bragg gratings at nominally equal wavelengths." Appl. Opt., 37, 1741 (1998) 